\title{
Short stature in Korean women: a contribution to the multifactorial predisposition to gestational diabetes mellitus
}

\author{
H.C.Jang ${ }^{1}$, H.K.Min ${ }^{1}$, H.K.Lee ${ }^{2}$, N.H.Cho ${ }^{3}$, B.E.Metzger ${ }^{4}$ \\ ${ }^{1}$ Department of Medicine, Samsung Cheil Hospital, College of Medicine, Sung Kyun Kwan University, Seoul, Korea \\ ${ }^{2}$ Department of Medicine, College of Medicine, Seoul National University, Seoul, Korea \\ ${ }^{3}$ Department of Preventive Medicine, Ajou University Medical School, Suwon, Korea \\ ${ }^{4}$ Department of Medicine, Center for Endocrinology, Metabolism, and Molecular Medicine, Northwestern University Medical \\ School, Chicago, Illinois, USA
}

Summary We examined the associations between demographic characteristics including short stature and the prevalence of gestational diabetes mellitus (GDM) in Korean women. In this study, a total of 9005 pregnant women underwent universal screening for GDM. Oral glucose tolerance tests (100 g OGTT) were performed in positive screenees ( $1 \mathrm{~h}$ plasma glucose $\geq 7.2 \mathrm{mmol} / \mathrm{l}$ ) and GDM was diagnosed using National Diabetes Data Group criteria. Women with GDM were older and heavier than those with a positive screen and normal OGTT, as well as those with a negative screen. However, height of women with GDM was significantly shorter than those with a positive screen and normal OGTT, and a negative screen. When the study subjects were stratified according to height quartiles, the plasma glucose at the screening test decreased as height increased. Furthermore, the prevalence of GDM was highest in the shortest quartile $(\leq 157 \mathrm{~cm})$ group; the odds ratio for GDM was two times greater compared with the highest quartile ( $\geq 163 \mathrm{~cm}$ ) group, even after controlling for age and body mass index (BMI). In addition, multiple logistic regression analysis revealed that greater prepregnancy BMI, age, weight gain, a parental history of diabetes mellitus, and shorter maternal height were directly and independently associated with the prevalence of GDM. We have found that short stature is an independent risk factor for GDM in the racially homogenous population of Seoul, Korea. It is suggested that this propensity may be conveyed primarily by environmental influences. However, genetic factors may also modify the response to the environmental insult. Our findings also emphasize the heterogeneity of factors which predispose to GDM. [Diabetologia (1998) 41: 778-783]

Keywords Short stature, gestational diabetes mellitus, prevalence, heterogeneity.
Gestational diabetes mellitus (GDM), which is defined as carbohydrate intolerance of variable severity with onset or first recognition during the present pregnancy [1], is associated with adverse outcomes

Received: 15 December 1997 and in revised form: 16 February 1998

Corresponding author: Hak C. Jang, MD, Department of Medicine, Samsung Cheil Hospital, College of Medicine, Sung Kyun Kwan University, 1-19, Mookcheong-Dong, Chung-Ku, Seoul 100-380, Korea

Abbreviations: GDM, Gestational diabetes mellitus; IGT, impaired glucose tolerance; OGTT, oral glucose tolerance test; SGA, small for gestational age; AGA, average for gestational age. of pregnancy including macrosomia, birth trauma and metabolic complications of the newborn [2-4]. Although most women with GDM have a normal glucose tolerance when tested several weeks postpartum, impairment of insulin secretory capacity or increased insulin resistance or both have been reported in these women $[5,6]$, and a substantial proportion of the women with previous GDM will eventually develop Type II diabetes mellitus [7-9]. The reported prevalence of GDM has ranged widely from less than $1 \%$ to more than $10 \%$ [10]. To some extent, this wide variation is thought to reflect a lack of a standardization of screening test procedures and diagnostic criteria of GDM. However, it may also be attributed to the heterogeneous nature of GDM with respect to ethnic or racial influence or both, genetic factors, admixture 
of subjects at risk for Type II diabetes mellitus, maternal age, and anthropometric characteristics [11-13].

Epidemiological studies in Hertfordshire and Preston (UK) [14, 15] reported that subjects 46 to 70 years of age who had lower birthweight and weight at 1 year of age had a higher prevalence of impaired glucose tolerance (IGT) or Type II diabetes mellitus or both. The authors of these studies suggested that impaired nutrition or growth during fetal development and early infancy or both were associated with shorter stature and increased risk of IGT or Type II diabetes mellitus or both in adult life. We reasoned that if such associations occur in pregnant women, an association might be seen between women's height and prevalence of GDM.

To examine this hypothesis, we analysed the relationship between demographic characteristics and the prevalence of GDM in pregnant Korean women. This study was a large-scale hospital-based cohort that has undergone the standardized screening and diagnostic method as recommended by the 3rd International Workshop-Conference on GDM [1]. We modified this protocol only by the use of a $7.2 \mathrm{mmol} / \mathrm{l}$ threshold for a positive screen instead of $7.8 \mathrm{mmol} / \mathrm{l}$ to increase the sensitivity of the screening procedure [13].

\section{Subjects and methods}

Subjects. The study included consecutive pregnant Korean women who received antenatal care at the Samsung Cheil Hospital from 1 January 1991 to 30 November 1994. Samsung Cheil Hospital is a community hospital serving women of diverse socioeconomic status living in Seoul metropolitan area. Since 1 January 1991, all pregnant women have been screened for glucose intolerance at 24 to 28 weeks' gestation. Pregnant women who did not receive antenatal care until after 28 weeks' gestation were screened as soon as possible, but women who were referred because of having high risk factors or transferred from other hospitals were not included in this study. Pregnant women known to have diabetes mellitus, a history of previous GDM, or a medical disease that affects glucose metabolism were excluded. During the study period, 9005 consecutive women underwent screening for GDM. The study was approved by the Samsung Cheil Hospital research and ethics committee.

Screening and diagnostic test. The screening test was performed at any time of day without regard to time or size of the last meal. Women with a positive screen (plasma glucose $\geq 7.2 \mathrm{mmol} / \mathrm{l}$ ) were recalled within 2 weeks for a $3 \mathrm{~h}, 100-\mathrm{g}$ oral glucose tolerance test (OGTT). The methods of screening and diagnostic testing were described in detail $[3,12]$ in our previous report. Patients were considered to have GDM if at least two values reached or exceeded the following: $5.8 \mathrm{mmol} / \mathrm{l}$ at fasting, $10.6 \mathrm{mmol} / \mathrm{l}$ at $1 \mathrm{~h}, 9.2 \mathrm{mmol} / \mathrm{l}$ at $2 \mathrm{~h}, 8.1 \mathrm{mmol} / \mathrm{l}$ at $3 \mathrm{~h}[16]$.

Prepregnancy weight was recorded from patient's recall, and height and body weight were measured at the time of the screening test. Height was measured to the nearest centimeter without shoes using FA-94H height scale (Fanics, Korea) and body weight was measured to the nearest $0.1 \mathrm{~kg}$ wearing light clothing. Measurements were taken by one observer. When a patient's recall of her prepregnancy weight was uncertain, weight at the first prenatal registration (before 14 weeks' postconception) was substituted. The body mass index (BMI; kg/ $\mathrm{m}^{2}$ ) was calculated from height and prepregnancy weight. Obesity was defined as prepregnancy BMI of 27.3 or more. A history of diabetes mellitus in parents was limited to those who were diagnosed as having diabetes mellitus by the measurement of blood glucose concentration and treated with oral hypoglycaemic agents or insulin.

Plasma glucose concentration was measured by a glucose oxidase method using a YSI 2300 STAT (Yellow Springs Instrument Co., Yellow Springs, USA). The concentration of insulin was measured in duplicate, using commercially available kits (Dainabot Co., Tokyo, Japan). The intra-assay and interassay coefficients of variation for insulin measurements were 4.5 and $8.8 \%$.

Statistical analysis. Data are expressed as means \pm SD and percentage, unless otherwise stated. The differences of normally distributed variables among groups were analysed by analysis of variance (ANOVA) and post-Hoc (Duncan's multiple range) test, and analysis of covariance (ANCOVA) test. Log transformation of insulin concentration was applied to correct for non-normal distributions. Kruskal-Wallis and chi-square tests were also used to analyse not normally distributed and dichotomous variables (e.g. \% obesity, parental history of diabetes mellitus, and parity). Correlation between study variables was tested by Pearson or Spearman correlation. The effect of demographic characteristics on the prevalence of GDM was evaluated by multiple logistic regression analysis. All analyses were performed with SPSS for Windows (Chicago, Ill., USA).

\section{Results}

Of the 9005 pregnant women who were screened for GDM, $2050(22.8 \%)$ had a positive screen. However, a small group of 142 failed to complete the OGTT because of lack of compliance or vomiting after ingestion of the glucose solution and were excluded from statistical analysis. Therefore, a total of 1908 (93.1\%) underwent $3 \mathrm{~h}, 100 \mathrm{~g}$ OGTT. Of the 1908 who completed $3 \mathrm{~h}$ OGTT, 173 pregnant women were classified as having GDM. Therefore, the overall prevalence of GDM amongst them was 1.9 cases per 100 screened.

Demographic characteristics of women with GDM were compared with the women with a negative screen and women with a positive screen and a normal OGTT (Table 1). Women with GDM were older and heavier than the positive screen and normal OGTT and also the negative screen groups. The frequency of obesity was highest in the GDM group and lowest in the negative screen group. Obesity was present in only $1.4 \%$ of all the women but $9.8 \%$ of those with GDM. On the other hand, height of the women was inversely related to glycaemia with a decrease in height across the three groups from the screen negative to GDM group. The frequency of a positive parental history of diabetes mellitus in the 
Table 1. Demographic characteristics of study subjects

\begin{tabular}{|c|c|c|c|}
\hline & $\begin{array}{l}\text { Negative screen } \\
(n=6,955)\end{array}$ & $\begin{array}{l}\text { Positive screen, normal OGTT } \\
(n=1,735)\end{array}$ & $\begin{array}{l}\text { GDM } \\
(n=173)\end{array}$ \\
\hline Age (yr.) & $28.5 \pm 3.4$ & $29.4 \pm 3.5^{\mathrm{a}}$ & $31.1 \pm 4.2^{\mathrm{ac}}$ \\
\hline $\begin{array}{l}\text { Prepregnancy } \\
\text { weight }(\mathrm{kg}) \\
\text { BMI }\left(\mathrm{kg} / \mathrm{m}^{2}\right) \\
\% \text { Obesity }(\mathrm{BMI} \geq 27.3)\end{array}$ & $\begin{array}{l}51.6 \pm 6.4 \\
20.1 \pm 2.3 \\
1.0 \%\end{array}$ & $\begin{array}{l}52.4 \pm 7.2^{\mathrm{a}} \\
20.7 \pm 2.7^{\mathrm{a}} \\
2.4 \%^{\mathrm{a}}\end{array}$ & $\begin{array}{l}56.5 \pm 9.5^{\mathrm{ac}} \\
22.6 \pm 3.4^{\mathrm{ac}} \\
9.8 \% \text { a c }\end{array}$ \\
\hline $\begin{array}{l}\text { Screening test } \\
\text { weight } \\
\text { weight gain }\end{array}$ & $\begin{array}{r}59.7 \pm 7.0 \\
8.1 \pm 3.1\end{array}$ & $\begin{aligned} 60.9 & \pm 7.7^{\mathrm{a}} \\
8.3 & \pm 3.3\end{aligned}$ & $\begin{aligned} 64.9 & \pm 10.1^{\mathrm{ac}} \\
8.4 & \pm 3.9\end{aligned}$ \\
\hline Height $(\mathrm{cm})$ & $160.0 \pm 4.2$ & $159.0 \pm 4.6^{\mathrm{a}}$ & $158.1 \pm 4.9^{\mathrm{ac}}$ \\
\hline Parental History of diabetes mellitus & $13.2 \%$ & $17.6 \%^{\mathrm{a}}$ & $30.1 \%$ a c \\
\hline 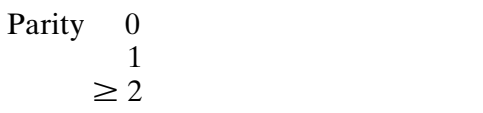 & $\begin{array}{r}66.3 \% \\
31.1 \% \\
2.6 \%\end{array}$ & $\begin{array}{c}62.7 \% \\
32.6 \% \\
4.7 \% \mathrm{a}\end{array}$ & $\begin{array}{l}55.5 \%^{\mathrm{b}} \\
34.7 \% \\
9.8 \%^{\text {a d }}\end{array}$ \\
\hline
\end{tabular}

Negative screen: women with screening test glucose value $<7.2 \mathrm{mmol} / \mathrm{l}$, positive screen, normal OGTT: women with screening glucose value $\geq 7.2 \mathrm{mmol} / \mathrm{l}$ and a normal glucose tolerance test.

${ }^{\mathrm{a}} p<0.001,{ }^{\mathrm{b}} p<0.01$ compared with negative screen, ${ }^{\mathrm{c}} p<0.001,{ }^{\mathrm{d}} p<0.01$ compared with positive screen, normal OGTT

Table 2. Plasma glucose and insulin concentrations during oral glucose tolerance test in women with a positive screen according to height quartile

\begin{tabular}{|c|c|c|c|c|c|}
\hline & \multicolumn{4}{|c|}{ Height quartile } & \multirow[t]{2}{*}{$\mathrm{P}$} \\
\hline & $\begin{array}{l}\leq 157 \mathrm{~cm} \\
(n=655)\end{array}$ & $\begin{array}{l}159 \mathrm{~cm} \\
(n=341)\end{array}$ & $\begin{array}{l}162 \mathrm{~cm} \\
(n=492)\end{array}$ & $\begin{array}{l}\geq 163 \mathrm{~cm} \\
(n=420)\end{array}$ & \\
\hline \multicolumn{6}{|c|}{ Glucose (mmol/l) } \\
\hline Fasting & $4.38 \pm 0.59$ & $4.36 \pm 0.68$ & $4.39 \pm 0.53$ & $4.39 \pm 0.67$ & NS \\
\hline $1 \mathrm{~h}$ & $8.25 \pm 1.83$ & $8.36 \pm 1.89$ & $8.08 \pm 1.81$ & $8.08 \pm 1.90$ & 0.09 \\
\hline $2 \mathrm{~h}$ & $7.38 \pm 1.79$ & $7.43 \pm 1.81$ & $7.26 \pm 1.76$ & $7.12 \pm 1.87$ & 0.06 \\
\hline $3 \mathrm{~h}$ & $6.64 \pm 1.37$ & $6.64 \pm 1.40$ & $6.48 \pm 1.50$ & $6.22 \pm 1.55$ & $<0.001$ \\
\hline \multicolumn{6}{|c|}{ Insulin (pmol/l) } \\
\hline Fasting & $39 \pm 20$ & $39 \pm 20$ & $38 \pm 20$ & $41 \pm 22$ & NS \\
\hline $1 \mathrm{~h}$ & $336 \pm 194$ & $341 \pm 184$ & $338 \pm 208$ & $339 \pm 198$ & NS \\
\hline $2 \mathrm{~h}$ & $344 \pm 223$ & $351 \pm 205$ & $338 \pm 227$ & $338 \pm 229$ & NS \\
\hline $3 \mathrm{~h}$ & $287 \pm 175$ & $288 \pm 186$ & $268 \pm 178$ & $247 \pm 158$ & $<0.005$ \\
\hline
\end{tabular}

GDM group was twice as high as that in the negative screen group. The proportion of women with a parity 2 or more was higher in the GDM group than those in the positive screen and normal OGTT and negative screen groups.

We stratified all the study subjects into height quartiles for further delineation of the relationship between height and glycaemia in gestational diabetes. However, the number of subjects per quartiles were not identical because we measured the height to the nearest centimeter. Figure 1 illustrates glucose challenge test values according to height quartiles after adjustment for age and BMI. The ageand BMI-adjusted glucose challenge test values were highest in the shortest quartile group and these values decreased significantly as the height increased. The frequency of a positive screen also decreased as the height increased $(\leq 157 \mathrm{~cm}, 28.6 \%$; $159 \mathrm{~cm}, 21.0 \% ; 162 \mathrm{~cm}, 19.0 \% ; \geq 163 \mathrm{~cm}, 17.8 \%$, $p<0.001)$.
Plasma glucose and insulin concentrations during the OGTT in women with a positive screen are listed in Table 2. The fasting $1 \mathrm{~h}$ and $2 \mathrm{~h}$ glucose concentrations were not different among height quartile groups but showed a decreasing tendency of 1 and $2 \mathrm{~h}$ glucose concentrations as height increased. However, $3 \mathrm{~h}$ glucose concentration significantly increased as height decreased. Similar relationships were found between height and insulin concentrations during the OGTT. The $3 \mathrm{~h}$ insulin concentration decreased as height increased. Height and $3 \mathrm{~h}$ glucose and insulin concentrations were inversely correlated ( $r=0.13, p<0.001, r=0.11, p<0.001$, respectively).

Table 3 shows the raw prevalence of GDM, the age- and BMI-adjusted prevalence of GDM and odds ratio for GDM according to height stratified by quartiles. There was an increase in prevalence of GDM with decreasing height $(p<0.001)$. The prevalence of GDM was $3.2 \%$ in the shortest quartile group of height and the highest quartile group 


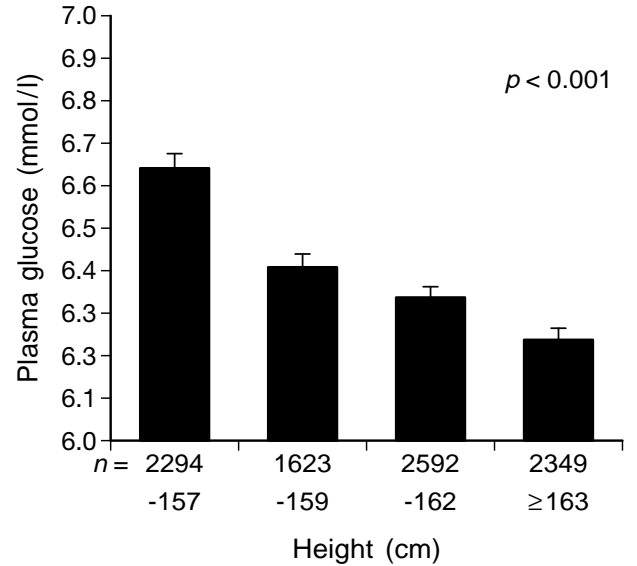

Fig. 1 Means \pm SEM values of glucose challenge test according to height quartiles after adjustment for age and BMI. The ageand BMI-adjusted values of glucose challenge test were highest in the shortest height quartile, and decreased significantly as the height increased
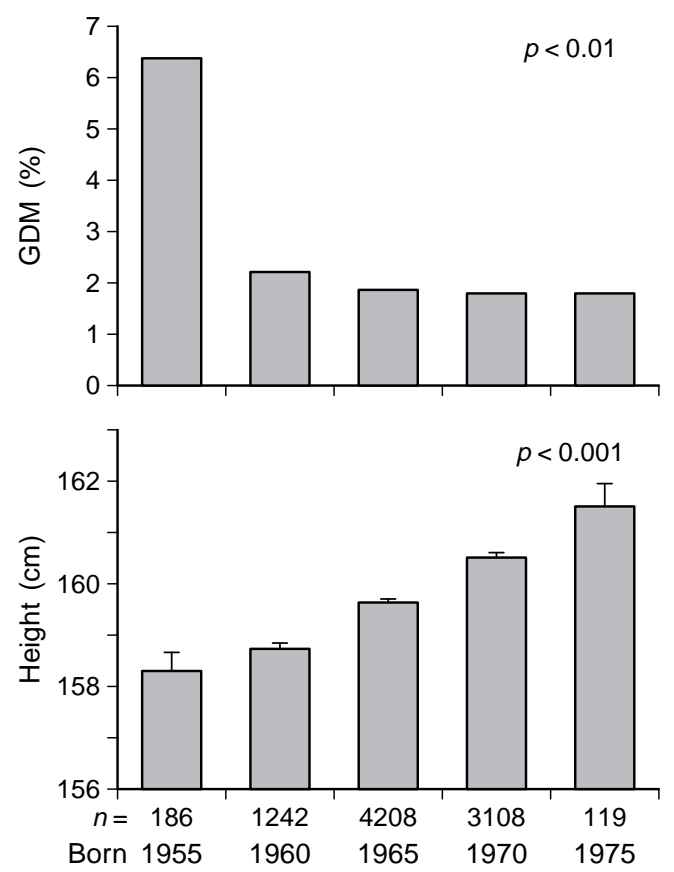

Fig. 2 Age-adjusted prevalence of GDM and height (means \pm SEM) of each 5-year birth cohort. The age-adjusted prevalence of GDM was highest in the women born in 1955 and decreased rapidly as birth cohort increased. Women's heights were least in those born in 1955 and greatest in those born in 1975

showed $1.2 \%$. Because the shortest quartile group was older in age with higher BMI than the other height quartiles, we compared the prevalence and odds ratio of GDM among height quartiles after adjustment for age and BMI. As shown in Table 3, an inverse trend association between the height and prevalence of GDM was persistent even after adjusting for age and BMI $(p<0.001)$. The age- and BMI-
Table 3. Prevalence and odds ratio for gestational diabetes mellitus: maternal height stratified by quartile

\begin{tabular}{lllllll}
\hline $\begin{array}{l}\text { Height quartile } \\
(\mathrm{cm})\end{array}$ & $n$ & \multicolumn{2}{c}{ GDM } & $\begin{array}{c}\text { Odds } \\
\text { ratio }^{\mathrm{a}}\end{array}$ & 95\% CI \\
\cline { 3 - 5 }$\geq 163$ & 2349 & 29 & 1.2 & 1.5 & 1.00 & \\
162 & 2592 & 39 & 1.5 & 1.6 & 1.15 & $0.66-2.03$ \\
159 & 1628 & 32 & 2.0 & 2.1 & 1.50 & $0.91-2.46$ \\
$\leq 157$ & 2294 & 73 & 3.2 & 2.8 & 2.04 & $1.37-3.03$ \\
\multicolumn{1}{l}{$\begin{array}{l}\text { Total population } \\
160\end{array}$} & & & & & \\
\hline
\end{tabular}

${ }^{a}$ Age and BMI adjusted prevalence of GDM and odds ratio

Table 4. Multiple logistic regression $\operatorname{model}^{\mathrm{a}}$ for gestational diabetes mellitus

\begin{tabular}{lccl}
\hline Variable & Coefficient & SEM & Significance \\
\hline Prepregnancy BMI & 0.2367 & 0.0240 & $<0.0001$ \\
Age & 0.1163 & 0.0196 & $<0.0001$ \\
Parental history of & & & \\
diabetes mellitus & 0.4329 & 0.0868 & $<0.0001$ \\
Height & -0.0688 & 0.0179 & $<0.0001$ \\
Weight gain & 0.0659 & 0.0225 & $<0.005$ \\
\hline
\end{tabular}

a Included Variables: age, prepregnancy weight, prepregnancy BMI, height, parental history of diabetes mellitus, parity, and weight gain until screening test

adjusted odds ratio in the shortest height quartile was twofold higher than that in the highest height quartile group.

Multiple logistic regression analysis was performed to identify significant and independently associated factors such as height for GDM. The variables included in the multivariate analysis were age, prepregnancy weight, prepregnancy BMI, height, parity, parental history of diabetes mellitus, and weight gain during pregnancy until the time of the screening test. The list of independent variables in order of significance is presented in Table 4. Prepregnancy BMI was the first variable entered into the model. The women's ages, parental history of diabetes mellitus, and weight gain during gestation were also independently associated with GDM; however, prepregnant weight and parity were not. Height also remained as a significant and independent factor.

In this study, we have little information on potentially confounding variables that influence adult height such as genetics, size at birth, nutrition during fetal life and infancy, and general health (infection, etc.) during childhood. However, we observed an overall correlation between age and height. Therefore, we plotted height of the birth cohort grouped by 5 year intervals covering the period from 1950 to 1975. Figure 2 depicts the age-adjusted prevalence of GDM and the height at each 5-year birth cohort group. The age-adjusted prevalence of GDM in wom- 
en born in 1955 was highest, 6.4\%, however, height was shortest in these women. The age-adjusted prevalence of GDM decreased rapidly as the birth cohort increased. However, there was no decrease in prevalence of GDM among women born after 1965. The height was shortest in women born in earlier years, (1955 birth cohort) and tallest in the 1975 birth cohort. Height did not differ in the 1955 and 1960 birth cohort groups. However, height increased significantly in each succeeding cohort after 1960.

\section{Discussion}

Freinkel introduced a novel way of thinking about "fuel-mediated teratogenesis" in his 1980 Banting Lecture [17]. He proposed that the nature and severity of the possible long-term implications on the offspring of disturbed intrauterine metabolism were linked to the period of pregnancy when these disturbances occurred and to the state of development of the cells potentially affected by the changes in intrauterine metabolism.

Recent epidemiologic studies in the United Kingdom have indicated that those with a lower birth weight, small for dates or of lower weight during early infancy had increased rates of cardiovascular disease and impaired glucose tolerance (IGT) or Type II diabetes mellitus or both as adults $[14,15,18]$. Among 140 men and 126 women born in Preston (UK) between 1935 and 1943 whose size at birth had been measured in detail, the percentage who had Type II diabetes mellitus or IGT fell progressively from $27 \%$ in those whose birth weight was $2.50 \mathrm{~kg}$ or less to $6 \%$ in those whose was $3.41 \mathrm{~kg}$ or more [15]. Plasma glucose concentrations at $2 \mathrm{~h}$ in the OGTT fell as birth weight increased, as did $2 \mathrm{~h}$ plasma insulin concentration. These associations appear to be related to impaired intrauterine growth rather than prematurity and independent of current obesity and fat distribution. The authors also pointed out that those who had lower birth weights were shorter and lighter, but their BMI was similar to that of other subjects. A relationship between the low birthweight and glucose intolerance has also been described in young Mexican-American adults and Pima Indians [19, 20].

In this study, we found that pregnant Korean women with GDM were significantly shorter than those with normal glucose tolerance during pregnancy. The age and BMI-adjusted prevalence of GDM increased from $1.5 \%$ in the highest height quartile group to $2.8 \%$ in the shortest height quartile group. The plasma glucose concentration measured at the glucose challenge test decreased as maternal height increased. Furthermore, multiple logistic regression analysis indicated that there was an independent and inverse relationship between height and GDM. However, we were not able to examine the relationship between the women's birth weights and prevalence of GDM or plasma glucose concentration, because many of our study subjects, especially in early birth cohorts, had been delivered at home and such data are not available. Therefore, we stratified the study group according to 5-year intervals based on the birth years. As shown in Figure 2, the age-adjusted prevalence of GDM decreased and the mean height increased across the 5-year birth cohort groups.

A number of previous reports indicate that height is determined by several important factors including genetics, intrauterine environment (time of onset or severity of intrauterine growth retardation) and postnatal growth (nutrition, infection, etc.) [21]. Several follow-up studies [22, 23] of small-for-gestationalage (SGA) children suggest that the short stature may persist in childhood and later life. These studies described that rates of weight gain, length and head circumference in the SGA infants were higher than those in average-for-gestational-age (AGA) infants during the first 6 months after birth. However, after this period, there were no consistent differences in growth rates between SGA and AGA infants. On average, SGA infants increase their weight relatively more than length, but they are still shorter and lighter than AGA infants at the end of the first year. Fancourt et al. [22] suggested that postnatal growth in SGA infants depended on severity and time of onset of intrauterine growth retardation. Children whose onset of growth retardation started before 34 weeks of gestation were shorter and lighter than those whose onset of growth retardation started after 34 weeks. Westwood et al. [23] also demonstrated a persistent deficit in height among SGA cohort without known causes for low birth weight (e.g., congenital anomalies, intrauterine infection, chromosomal abnormalities, or perinatal asphyxia) in adolescence (age 13 to 19 years), even after controlling for parental height.

In our study, the trend toward an increase in the women's heights was very similar to that of the increase in gross national product (GNP) in Korea (1955, 1.4 billion dollar; 1960, 1.8 billion dollar; 1965, 2.7 billion dollar; 1970, 5.6 billion dollar; 1975, 14.7 billion dollar; [24]). After 1960, there was a rapid increase in height that was parallel to GNP increase. Thus, the height of Korean women might be significantly affected by the economic status of Korea.

We are aware of two hospital-based prospective studies $[2,25]$ in North America which reported that pregnant women with GDM were significantly shorter than those with normal glucose tolerance. However, the implication of short stature of pregnant women in the GDM was not examined. Recently, Brown et al. [26] in Ely (UK) showed that men and women with IGT were significantly shorter than control subjects matched for sex, age, and BMI. Plasma glucose 
at $2 \mathrm{~h}$ was inversely correlated to height in men and women. However, this relationship was not significant in women after controlling for age and BMI.

In conclusion, we have found short stature is an independent risk factor for GDM in the racially homogenous population of Seoul, Korea. The changes in stature and prevalence of GDM appear to be conveyed by environmental influences. However, genetic factors may well modify the response to the environmental insult. Our findings also emphasize the heterogeneity of factors which predispose to GDM, as well as other forms of glucose intolerance.

Acknowledgements. We thank Drs. H. Y. Chung, I. K. Han in Medicine; Drs. E.S. Kim, M. Y. Kim, H. M. Ryu in Obstetrics; M.S. Kim, M. H. Park and other staff of the Endocrine Laboratory, all from Samsung Cheil Hospital. The study was supported, in part, by a Seulwon Grant from the Korean Diabetes Association and a Research Grant from the Cheil Medical Foundation.

Presented in part at the 55th Annual Meeting \& Scientific Sessions of American Diabetes Association, Atlanta, Georgia, 10-13 June, 1995.

\section{References}

1. Metzger BE (1991) Summary and recommendations of the Third International Workshop-Conference on Gestational Diabetes Mellitus. Diabetes 40 [Suppl 2]:197-201

2. Jacobson JD, Cousins L (1989) A population-based study of maternal and perinatal outcome in patients with gestational diabetes. Am J Obstet Gynecol 161: 981-986

3. Jang HC, Cho NH, Min YK, Han IK, Jung KB, Metzger BE (1997) Increased macrosomia and perinatal morbidity independent of maternal obesity or advanced maternal age in Korean women with GDM. Diabetes Care 20: $1582-1588$

4. Langer O, Levy J, Brustman L, Anyaegbunam A, Merkatz $\mathrm{R}$, Divon M (1989) Glycemic control in gestational diabetes mellitus - How tight is tight enough: small for gestational age versus large for gestational age? Am J Obstet Gynecol 161: 646-653

5. Buchanan TA, Metzger BE, Freinkel N, Bergman RN (1990) Insulin sensitivity and B-cell responsiveness to glucose during late pregnancy in lean and moderately obese women with normal glucose tolerance or mild gestational diabetes. Am J Obstet Gynecol 162: 1008-1014

6. Ryan EA, Imes S, Liu D et al. (1995) Defects in insulin secretion and action in women with a history of gestational diabetes. Diabetes 44: 506-512

7. Metzger BE, Cho NH, Roston SM, Radvany R (1993) Prepregnancy weight and antepartum insulin secretion predict glucose tolerance five years after gestational diabetes mellitus. Diabetes Care 12: 1598-1605

8. O'Sullivan JB (1989) The Boston gestational diabetes studies: review and perspectives. In: Sutherland HW, Stowers
JM, Pearson DWM (eds) Carbohydrate Metabolism in Pregnancy and the New-born. Springer Verlag, London, pp 287-294

9. Kjos S, Peters R, Xiang A, Henry O, Montoro M, Buchanan $T$ (1995) Predicting future diabetes in Latino women with gestational diabetes. Diabetes 44: 586-591

10. Hadden DR (1985) Geographic, ethnic, and racial variations in the incidence of gestational diabetes. Diabetes 34 [Suppl 2]:8-12

11. Berkowitz GS, Lapinski RH, Wein R, Lee D (1992) Race/ ethnicity and other risk factors for gestational diabetes. Am J Epidemiol 135: 965-973

12. Jang HC, Cho NH, Jung KB, Oh KS, Dooley SL, Metzger BE (1995) Screening for gestational diabetes mellitus in Korea. Int J Gynaecol Obstet 51: 115-122

13. Dooley SL, Metzger BE, Cho N, Liu K (1991) The influence of demographic and phenotypic heterogeneity on the prevalence of gestational diabetes mellitus. Int J Gynaecol Obstet 35: 13-18

14. Hales CN, Barker DJP, Clark PMS, et al. (1991) Fetal and infant growth and impaired glucose tolerance at age 64 BMJ 303: 1019-1022

15. Phipps K, Barker DJP, Hales CN, Fall CHD, Osmond C, Clark PMS (1993) Fetal growth and impaired glucose tolerance in men and women. Diabetologia 36: 225-228

16. National Diabetes Data Group (1979) Classification and diagnosis of diabetes and other categories of glucose intolerance. Diabetes 28: 1039-1057

17. Freinkel N (1980) Banting Lecture 1980: of pregnancy and progeny. Diabetes 29: 1023-1035

18. Barker DJP, Gluckman PD, Godfrey KM, Harding JE, Owens JA, Robinson JS (1993) Fetal Nutrition and cardiovascular disease in adult life. Lancet 341: 938-941

19. Valdez R, Athens MA, Thompson GH, Bradshaw BS, Stern MP (1994) Birthweight and adult health outcomes in a biethnic population in the USA. Diabetologia 37: 624-631

20. McCance DR, Pettitt DJ, Hanson RL, Jacobsson LTH, Knowler WC, Bennett PH (1994) Birth weight and non-insulin dependent diabetes: thrifty genotype, thrifty phenotype, or surviving small baby genotype? BMJ 308: 942-945

21. Davies DP (1981) Growth of small-for-dates babies. Early Human Development 5: 95-105

22. Fancourt R, Campbell S, Harvey D, Norman AP (1976) Follow-up study of small-for-date babies. BMJ 1: 1435-1437

23. Westwood M, Kramer MS, Munz D, Lovett JM, Watters GV (1983) Growth and development of full-term nonasphyxiated small-for-gestational-age newborns: follow-up through adolescence. Pediatrics 71: 376-382

24. Korea National Statistical Office (1995) Review on progress of Korean development through statistics. National Statistical Office, Seoul

25. Magee MS, Walden CE, Benedetti TJ, Knopp RH (1993) Influence of diagnostic criteria on the prevalence of gestational diabetes and perinatal morbidity. JAMA 269: 609-615

26. Brown DC, Byrne CD, Clark PMS et al. (1991) Height and glucose tolerance in adult subjects. Diabetologia 34: $531-533$ 